\title{
Internet of Things: Design and Manufacture of Home Automation System with Web Server Based Arduino and Android
}

\author{
Nataniel Dengen, Reza Maulana Yusuf, Pohny
}

\begin{abstract}
Home automation is an automated system created specifically to facilitate the work of humans, especially at home. Home Automation connects electronic devices to each other in a home that is integrated using the internet network and allows it to be controlled via android smartphone and web client (server). On android smartphone installed applications that have been connected with Arduino microcontroller and Ethernet shield. In this research for controlling or retrieving data via Ethernet network the easiest is Arduino serve as server and we can request and arrange data through web client or android application then data will be sent using protocol User Datagram Protocol (UDP). So to control the electronic devices can be done from a considerable distance as long as connected to the internet.
\end{abstract}

Keywords: Client; Ethernet; Home Automation; Server; User Datagram Protocol (UDP).

\section{INTRODUCTION}

The Internet of Things (IoT) has become a hot topic to talk about lately. IoT is not only a concept that affects human life but how IoT can help facilitate human life. The fact that the availability of broadband Internet is getting cheaper, as well as the price of technology developer tools such as Arduino microcontroller module and other technology modules. This fact makes IoT the perfect thing to use.

Simply put, the Internet of Things is the basic concept that connects any device to each other. Internet of things that penetrate the electronics home users can facilitate people in doing various things [1]. Automation of all devices connected to the internet where the automation configuration can be adjusted easily without having to come to the location of the device [2]. For example, if we want to turn on and turn on the lights, fan / air conditioner, open and close the door remotely [3].

In this paper will be discussed about the design and manufacture of Home Automation System with Web Server as the control center. The home automation system is supported by an internet connection that connects between: android smartphone to microcontroller module which then electronic device as output.

\section{EXPERIMENTAL DESIGN}

Revised Version Manuscript Received on 10 September, 2019.

NatanielDengen,Department of Informatics Engineering, Mulawarman University, Samarinda, Indonesia.(Email: reza.bunta@ gmail.com)

Reza Maulana Yusuf,Department of Informatics Engineering, Mulawarman University, Samarinda, Indonesia.

Pohny,Department of Informatics Engineering, Mulawarman University, Samarinda, Indonesia.

\subsection{Conceptual framework}

The conceptual framework is a form of thinking framework that can be used as an approach to problem solving. Usually this research framework uses a scientific approach and shows the relationship between variables in the analysis process [4]. The conceptual framework is as follows:

\section{Collection of data and information.}

Referenced library in this case can be a scientific journal, text book, or information in the form of trusted articles listed on the internet.

\section{Design Prototype Design.}

The design prototype will be equipped with Arduino Uno R3 Microcontroller module, 4 garden lights, 1 room light, 1 dining room lamp, 1 living room lamp with PIR sensor, SG90 servo, 2 servo MM996 Towerpro Micro Servo, Ethernet shield, gas sensor LPG, Light sensor, Buzzer, Breadboard, IP Cam, fan, resistor, cable, $5 \mathrm{v}$ power supply.

In addition to the selection of modules - modules that will be used in the manufacture of prototypes, this stage is also done that is making Smart House Design in the form of visual.

\section{Design Interface Design.}

Interface design is the basic design of the application, the relay driver circuit and other components.

Application Creation, Component Circuits and Program Writing.

This Android Application and Writing Program uses the MIT Inventor App [5]. Component setups use commonly used electronics components. Arduino Program Writing aims to read and classify data sent through the application interface that has been made before. Writing program using Arduino IDE compiler and using $C$ language [6], [7].

\section{Implementation and Testing.}

The application, the set of components and writing programs that have been created and uploaded will then be tested and implemented to a prototype.

\subsection{Microcontroller as a controller}

Figure 1 show the Controller applications or interfaces designed in this writing are controllers or remote on the 


\section{INTERNET OF THINGS: DESIGN AND MANUFACTURE OF HOME AUTOMATION SYSTEM WITH WEB SERVER BASED ARDUINO AND ANDROID}

fundamental devices that exist in a home [8], [9], where the communication media used to connect between the android device to Arduino Uno microcontroller using Wi-Fi technology [3], [9]. Data processed by the application is then transferred via Wi-Fi to the Ethernet Shield connected to the microcontroller which in this case acts as the brain or the controller, then the microcontroller to deliver electrical current to the devices in accordance with the needs of the output used.

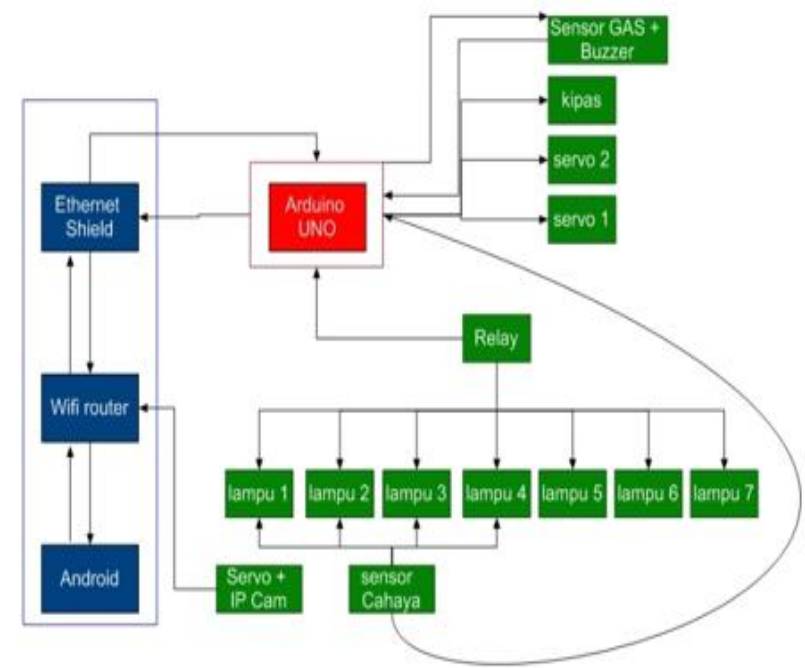

Fig. 1: Block diagram

\section{RESULT AND DISCUSSION}

In this study the authors designed a Smart House with Web Server and Full Keys where it can facilitate human activity when it wants to control electronic components that exist at home with android applications.

Control of electronic components is done by using android applications that have been designed, where data is sent to Arduino via Ethernet shield and Bluetooth shield will turn on or off electronic components in accordance with the needs or commands Figure 2 and Figure 3 show App display android created using MIT Inventor App 2.
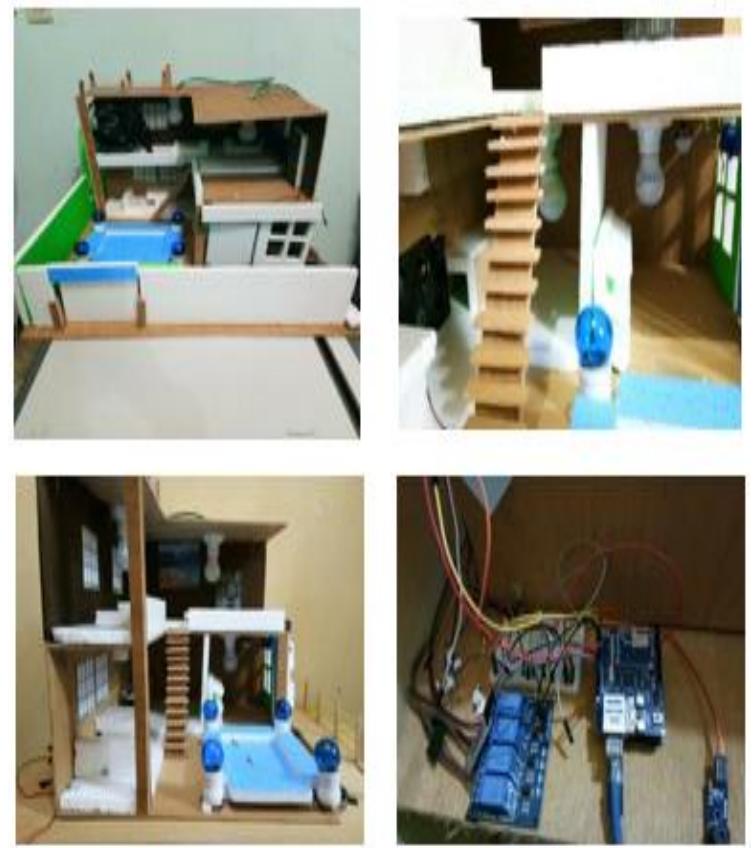

Fig. 2: Model prototype

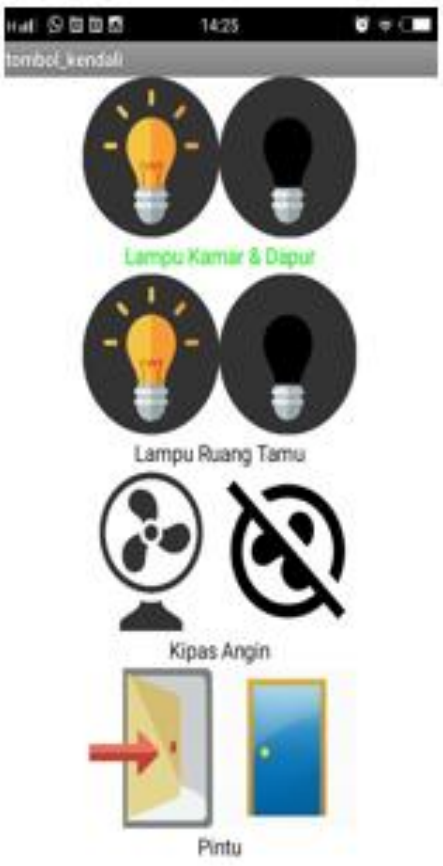

Fig. 3: Android app display

The server is in charge of coordinating all clients who are in their environment. The server that the author will create is a server that can control the electronic components and monitor the switch of each component whether it is in the ON or OFF position while taking the sensor data value, in this case the sensor MQ-06, the gas sensor butane, to then given to the client who requested. The protocol used is html, so we have to display it via the web. In order to access the smart home control panel the client must access the IP address owned by the server through the browser, so the browser can display the control panel that has been created using html shown in Figure 4.

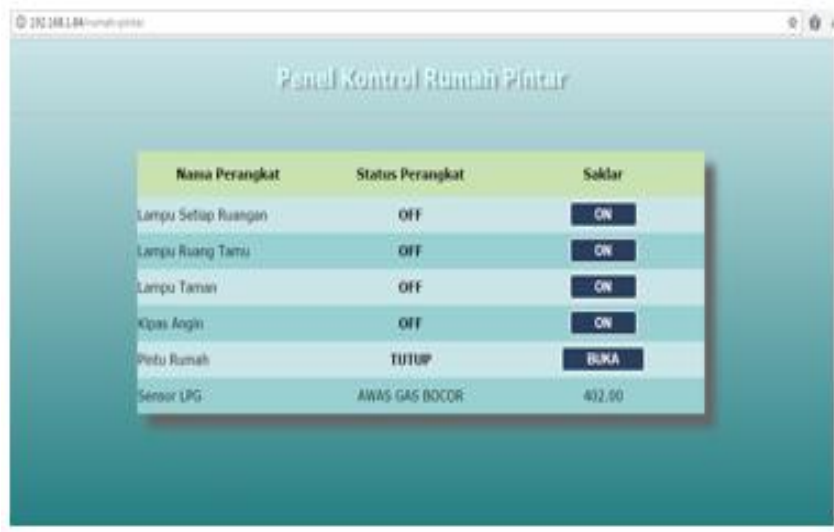

Fig. 4: Web view

\section{CONCLUSION}

After doing the system testing then it can be concluded from the overall performance of this Smart House system both in terms of hardware and software that users control the electronic components that exist at home using android applications remotely without having to switch to the location. For example, turn on and off the lights by using the

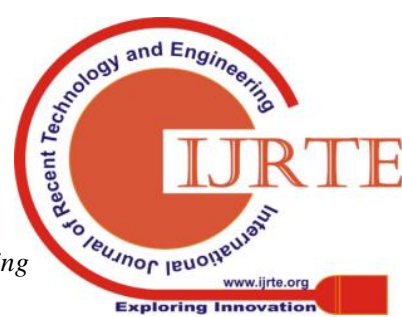


Android Application and Web Server. Android app will send Svoice commands using UDP protocol, so it can be read by Arduino and executed immediately. Each component has different delay. For example delay between servo 1 and servo 2 that is for 15 millisecond. Provide a sense of security to the user, because the smart home is equipped with a detector leakage. LPG gas. The system will instantly turn on Buzzer / Alarm after the sensor reaches more than 400 and Control distance up to 100 Meters.

\section{REFERENCES}

1. A. Wajiansyah, H. Purwadi, A. Astagani, and S. Supriadi, "Implementation of master-slave method on multiprocessor-based embedded system: case study on mobile robot," Int. J. Eng. Technol., vol. 7, no. 2, pp. 53-56, 2018.

2. S. Supriadi, A. Rizal, D. usilo B. Utomo, and A. Wajiansyah, "Line Follower Robot Optimization based Fuzzy Logic Controller Using Membership Function Tuning,” Int. J. Eng. Technol., vol. 7, no. 2, pp. 112-116, 2018.

3. V. A. Agaskar, A. Mithagari, A. Mhatre, and N. Shetty, "Internet of things: Home automation and surveillance system,” Int. Educ. Sci. Res. J., vol. 2, no. 4, pp. 41-43, 2016.

4. J. Chandramohan, R. Nagarajan, K. Satheeshkumar, N. Ajithkumar, P. A. Gopinath, and S. Ranjithkumar, "Intelligent smart home automation and security system using Arduino and Wi-fi,” Int. J. Eng. Comput. Sci., vol. 6, no. 3, pp. 20694-20698, 2017.

5. J. Gubbi, R. Buyya, S. Marusic, and M. Palaniswami, "Internet of Things (IoT): A vision, architectural elements, and future directions," Futur. Gener. Comput. Syst., vol. 29, no. 7, pp. 1645-1660, 2013.

6. R. Piyare, "Internet of things: ubiquitous home control and monitoring system using android based smart phone," Int. J. Internet Things, vol. 2, no. 1, pp. 5-11, 2013.

7. D. Kehagias and D. Nini, "Home Automation Based on an Android and a Web Application Using Raspberry Pi," Am. J. Mob. Syst. Appl. Serv., vol. 1, no. 3, pp. 174-181, 2015.

8. M. A. E.-L. Mowad, A. Fathy, and A. Hafez, "Smart Home Automated Control System Using Android Application and Microcontroller," Int. J. Sci. Eng. Res., vol. 5, no. 5, pp. 935-939, 2014.

9. R. Piyare and S. R. Lee, "Smart Home-Control and Monitoring System Using Smart Phone," in The 1st International Conference on Convergence and it's Application, 2013, pp. 83-86. 